\title{
Characterization of a hybrid GEM-Micromegas detector with respect to its application in a continuously read out TPC
}

\author{
Viktor Ratza ${ }^{1}$, Markus Ball ${ }^{1}$, M. Liebtrau ${ }^{1}$, and Bernhard Ketzer ${ }^{1}$ for the ALICE TPC Collaboration \\ ${ }^{1}$ Helmholtz-Institut für Strahlen- und Kernphysik, Nussallee 14-16, 53115 Bonn, Germany
}

\begin{abstract}
In the context of the upgrade of the LHC during the second long shutdown the interaction rate of the ALICE experiment will be increased up to $50 \mathrm{kHz}$ for $\mathrm{Pb}-\mathrm{Pb}$ collisions. As a consequence, a continuous read-out of the Time Projection Chamber (TPC) will be required. To keep the space-charge distortions at a manageable size, the ion backflow of the charge amplification system has to be significantly reduced. At the same time an excellent detector performance and stability of the system has to be maintained. A solution with four Gaseous Electron Multipliers (GEMs) has been adopted as baseline solution for the upgraded chambers. As an alternative approach a hybrid GEM-Micromegas detector consisting of one Micromegas (MM) and two GEMs has been investigated. The recent results of the study of the hybrid GEM-Micromegas detector will be presented and compared to measurements with four GEM foils.
\end{abstract}

\section{Introduction}

A Time Projection Chamber (TPC) [1] is used in many ongoing experiments like STAR [2] or ALICE [3] as central detector. With its low material budget and excellent pattern recognition capabilities it is an ideal device for three-dimensional tracking and identification of charged particles. The working principle of a TPC is based on ionization and gas amplification: Once a charged particle traverses the gas-filled detector the gas molecules will be ionized along the particle's trajectory. Owing to an electric field within the detector volume, the produced electrons will drift to the endplates where they get amplified and finally detected at a space-resolved readout. With a gas volume of $90 \mathrm{~m}^{3}$ the ALICE TPC is the largest TPC on the world. Up to now the readout is done by 72 MultiWire Proportional Chambers (MWPC) [4] with a total of 550000 readout cathode pads. In order to prevent ions to drift back to the active drift volume, a bipolar Gating Grid $(\mathrm{GG})$ is used which limits the theoretical readout rate to the order of $3 \mathrm{kHz}$. Together with the present readout system, the rate capability for central $\mathrm{Pb}-\mathrm{Pb}$ collisions is limited to $300 \mathrm{~Hz}$. As the interaction rate of the Large Hadron Collider (LHC) will be increased up to $50 \mathrm{kHz}$ for RUN3, the expected amount of events can not be handled by the current readout technology which demands a complete redesign of the TPC. In Ne- $\mathrm{CO}_{2}-\mathrm{N}_{2}$ (90-10-5) and at a total gain of 2000 the energy resolution must not exceed $\sigma / E=$ $12 \%$ for ${ }^{55} \mathrm{Fe}$ which translates to a $\mathrm{d} E / \mathrm{d} x$ resolution of about $5.5 \%$ and $7 \%$ in pp and central $\mathrm{Pb}-\mathrm{Pb}$ collisions. The ion backflow should be lower than $1 \%$ in order to be able to completely correct all effects from space-charge distortions [3]. Therefore the gated and MWPC-based readout technology will be replaced by a continuous read- out technology [5] based on Micro-Pattern Gaseous Detectors (MPGD). In previous investigations it has been shown that at least four GEM foils [6] with different pitches (see table 1) are required in order to cope with the aspired goal [7]. An alternative approach is given by the hybrid GEMMicromegas detector which consists of only two standard GEM foils and a bulk Micromegas [8].

\begin{tabular}{lccc}
\hline Geometry & $p / \mu \mathrm{m}$ & $d_{i} / \mu \mathrm{m}$ & $d_{o} / \mu \mathrm{m}$ \\
\hline small pitch (SP) & 90 & 40 & 60 \\
standard pitch (S) & 140 & 50 & 70 \\
medium pitch (MP) & 200 & 50 & 70 \\
large pitch (LP) & 280 & 50 & 70 \\
\hline
\end{tabular}

Table 1. Different GEM geometries ( $p$ pitch, $d_{i}$ inner diameter, $d_{o}$ outer diameter).

\section{Hybrid GEM-Micromegas detector}

Figure 1 shows the setup of the investigated hybrid GEMMicromegas detector: Due to the geometric and electrostatic structure of the Micromegas its ion backflow suppression capability is superior to a single GEM. The two GEM stages in front of the Micromegas are installed to keep the discharge probability of the device at a reasonable level while the energy resolution can be maintained. Furthermore the ion backflow can be reduced. Each standard GEM foil has a hexagonal hole structure according to table 1 and consists of a Polyimide substrate $(50 \mu \mathrm{m})$ which is coated with a thin layer of copper on both sides $(5 \mu \mathrm{m})$. To prevent unwanted overlapping effects of the holes, both foils are rotated by 90 degrees against each 


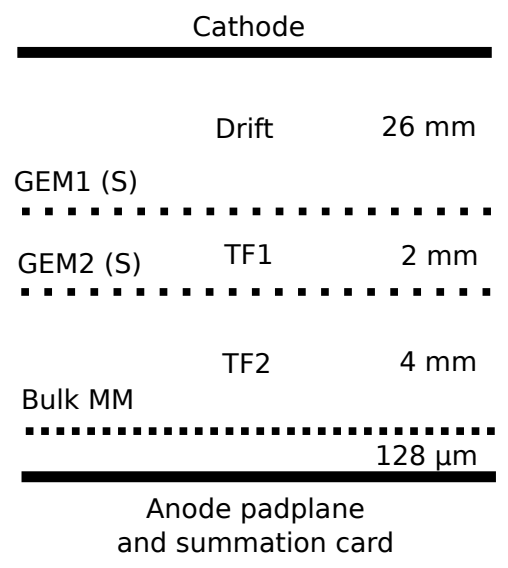

Figure 1. Schematic setup of the investigated hybrid GEMMicromegas detector.

other. The investigated bulk Micromegas was manufactured at CERN and has a gap of $128 \mu \mathrm{m}$ which separates a small mesh $(160$ lines $/ \mathrm{cm}$ ) from the readout padplane $(10 \mathrm{~cm} \times 10 \mathrm{~cm})$. Finally a summation card has been designed to sum up all 128 pads as four concentric rings. All rings can be read out individually or in an arbitrary combination. The setup has been assembled within a gas tight vessel with a volume of 6 liters. A Kapton window on the lid allows an external irradiation of the drift region with an $\mathrm{X}$-ray or an ${ }^{55} \mathrm{Fe}$ source. The currents at the cathode, the different GEM stages or the anode can be measured by picoammeters which were developed at the TU Munich [9] and which are sensitive for currents down to $20 \mathrm{pA}$ before systematic errors become dominant. In order to reduce electromagnetic influences and to shield the system, the whole detector and the picoammeters are placed in a Faraday cage made of copper and lead.

\section{Results}

\subsection{Gain contributions}

Depending on the field configuration above and below a GEM, electrons can get lost by drifting to the copper or the Kapton layer, i.e. the measured effective gain (equation 1) is a function of the field-dependent extraction- and collection efficiency $\epsilon_{\text {coll }}$ and $\epsilon_{\text {extr }}$ [10] of the GEM.

$$
G_{\mathrm{eff}}=\epsilon_{\mathrm{coll}} \cdot G_{\mathrm{abs}} \cdot \epsilon_{\mathrm{extr}}
$$

Finally the total gain of the detector can be expressed as the product of the effective gain of its individual amplification stages (equation 2).

$$
G_{\text {total }}=G_{\text {eff,GEM} 1} \cdot G_{\text {eff,GEM2 }} \cdot G_{\text {eff,MM }}
$$

In order to understand the contribution of the different amplification stages, the gain of each component has been measured. Figure 2 shows the absolute gain of the Micromegas as a function of the applied voltage: Since the ${ }^{55} \mathrm{Fe}$ source has a certain solid angle, the full padplane (all pads) needs to be read out in order to cover the avalanches

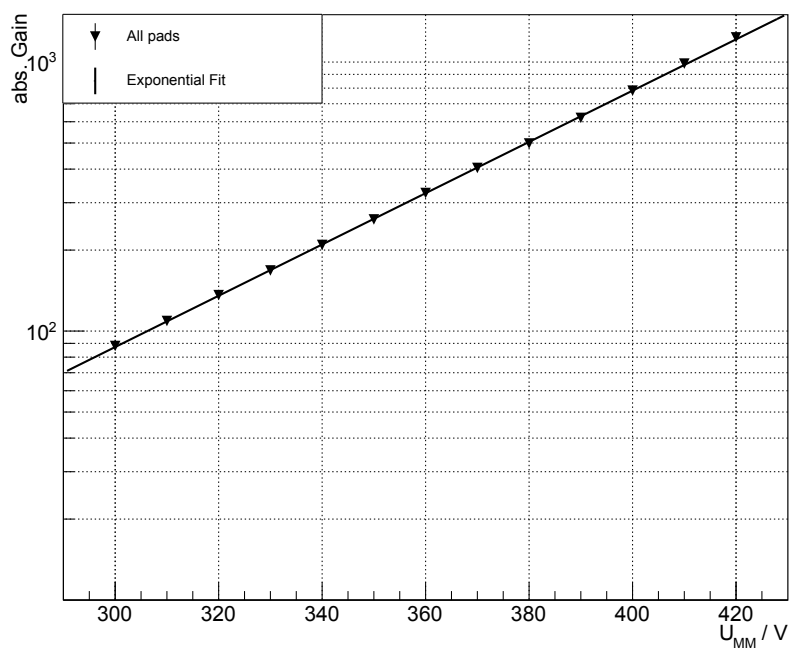

Figure 2. Absolute gain (=effective gain) of the Micromegas for different voltages $\left({ }^{55} \mathrm{Fe}\right.$ source in $\mathrm{Ne}-\mathrm{CO}_{2}(90-10)$, Drift $400 \mathrm{~V} / \mathrm{cm}, \mathrm{TF} 13 \mathrm{kV} / \mathrm{cm}$, TF2 $400 \mathrm{~V} / \mathrm{cm}$, GEM1 $250 \mathrm{~V}$, GEM2 $250 \mathrm{~V}$, Error x 5).

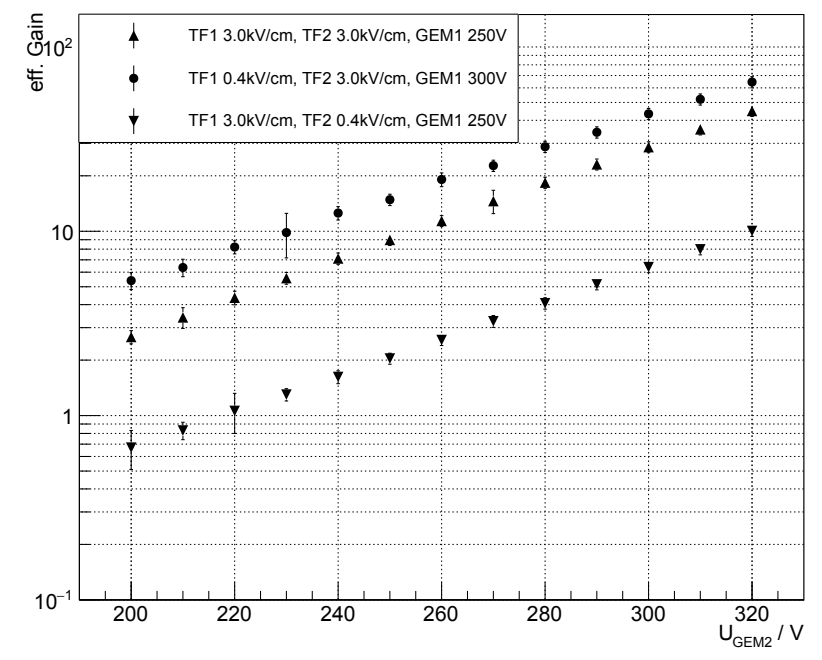

Figure 3. Effective gain of GEM2 for different field configurations $\left({ }^{55} \mathrm{Fe}\right.$ source in $\mathrm{Ne}-\mathrm{CO}_{2}(90-10)$, Drift $\left.400 \mathrm{~V} / \mathrm{cm}, \mathrm{MM} 0 \mathrm{~V}\right)$.

from all converted photons. The gain rises exponentially and is in the order of several $10^{2}-10^{3}$ for common Micromegas voltages. Since the primary charge above the first amplification stage is below the sensitive range of the picoammeters, the gain contribution of the first GEM can only be estimated by the second GEM (see figure 3): A field configuration of TF1 $0.4 \mathrm{kV} / \mathrm{cm}$ and TF2 $3.0 \mathrm{kV}$ is commonly used for the first amplification stage and thus represents the effective gain of the first GEM. Accordingly field configurations like TF1 $3.0 \mathrm{kV} / \mathrm{cm}$ and TF2 $0.4 \mathrm{kV} / \mathrm{cm}$ are common settings for the second GEM. Most of the total gain of the hybrid GEM-Micromegas detector is contributed by the Micromegas (order $10^{2}-10^{3}$ ), followed by the first GEM (order $10^{1}-10^{2}$ ). Commonly the second GEM is operated at an effective gain around 1 


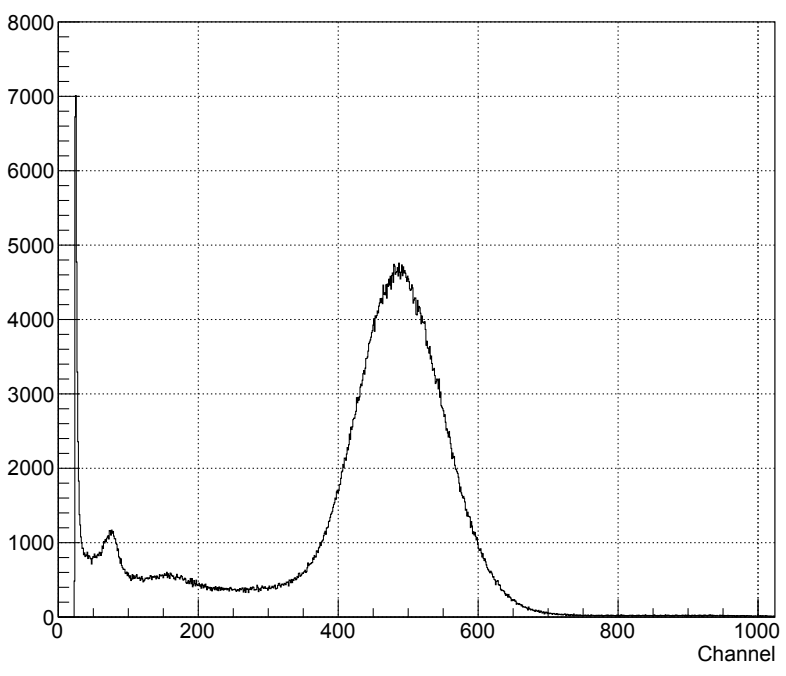

Figure 4. Example MCA spectrum for ${ }^{55} \mathrm{Fe}$ source in $\mathrm{Ne}-\mathrm{CO}_{2}$ (90-10) showing the central $\mathrm{K}_{\alpha / \beta}$ at $5.9 \mathrm{keV}$.

to maintain the energy resolution and to act as a further stage to block the ions from the Micromegas.

\subsection{Energy resolution and ion backflow}

For the planned upgrade of the ALICE TPC the ion backflow has to be less than $1 \%$ while the energy resolution $(\sigma / E)$ should not exceed $12 \%$ for ${ }^{55} \mathrm{Fe}$ (at a total gain of 2000 in $\mathrm{Ne}-\mathrm{CO}_{2}-\mathrm{N}_{2}(90-10-5)$ ) [3]. The energy resolution and the ion backflow of the hybrid GEM-Micromegas detector have been determined within a GEM1-Micromegas scan, i.e. the applied voltage of the first GEM was successively increased or decreased while the total gain of the detector was kept at a constant level of 2000 by adjusting only the voltage of the Micromegas. While the electrons from avalanches drift to the readout padplane, some of the produced ions may drift back to the cathode. Thus the ion backflow can be defined as the fraction of the currents at the readout padplane (anode) and the cathode according to equation 3 , where $\epsilon$ is the number of back drifting ions per incoming electron coming from the amplification region. For the ion backflow measurements an Amptek MiniX has been used to irradiate the detector $(30 \mathrm{kV}, 5 \mu \mathrm{A})$ and the whole padplane was read out.

$$
\mathrm{IB}=\frac{1+\epsilon}{G_{\text {eff }}}=\frac{I_{\text {cathode }}}{I_{\text {anode }}}
$$

All energy resolutions were determined with an ${ }^{55} \mathrm{Fe}$ source and the innermost pads $(2 \times 2)$ were read out. An example MCA spectrum is shown in figure 4: The prominent peak at the center belongs to $\mathrm{K}_{\alpha / \beta}$ photons which converted in the drift region while the lower energetic peaks appear due to conversion processes between the amplification stages (GEM1-GEM2 and GEM2-Micromegas). To determine the energy resolution the $\mathrm{K}_{\alpha / \beta}$ peak was fitted by a simple Gaussian. Due to the non-negligible background under the peak a more refined fit model will

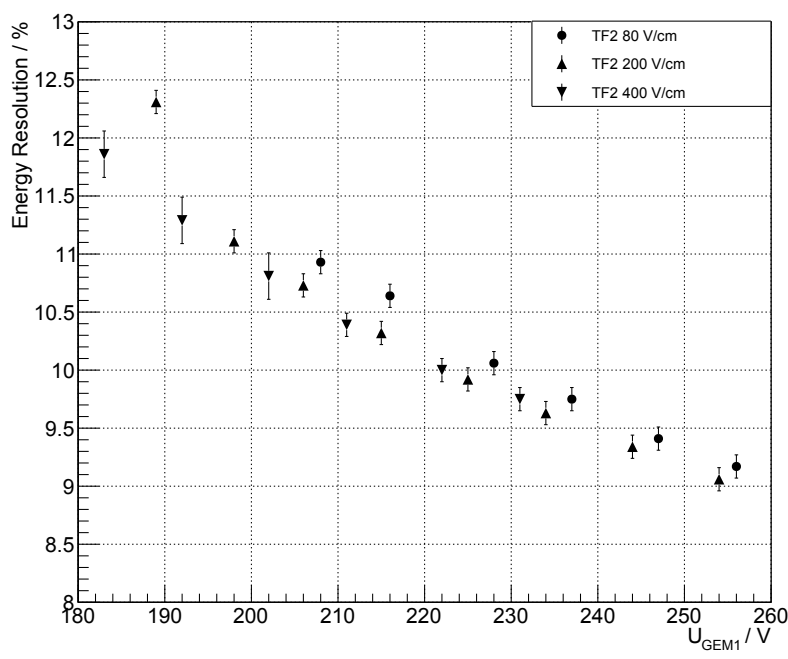

Figure 5. Energy resolution as a function of the GEM1 voltage (GEM1-MM scan in Ne-CO 2 (90-10), Drift $400 \mathrm{~V} / \mathrm{cm}$, TF1 $3 \mathrm{kV} / \mathrm{cm}$, GEM2 $250 \mathrm{~V}$, gain 2000, error energy resolution $\mathrm{x} 10$ )

be used in future investigations. Within the GEM1Micromegas scan the energy resolution of the detector can be tuned by changing the amplification at the first GEM stage as the number of avalanche electrons will change and influence the energy resolution statistics. Figure 5 shows that the energy resolution is mostly dominated by the first amplification stage (GEM1) and only weakly depends on TF2 or the Micromegas. Only for low TF2 $(80 \mathrm{~V} / \mathrm{cm})$ the energy resolution gets worse: Due to a small extraction efficiency of the second GEM electrons get lost within the transfer process to the Micromegas and hence its gain fluctuations become more dominant. Furthermore a change of the amplification at the first GEM strongly influences the ion backflow since the number of ions (which are immediately injected into the drift region) will be altered (figure 6 ). In contrast to the energy resolution the ion backflow is also influenced by TF2 and the Micromegas as most of the ions are produced within the last amplification stage (highest gain contribution). Thus the energy resolution and the ion backflow are not independent of each other: An optimization of the energy resolution leads to a degradation of the ion backflow and vice versa. Figure 7 illustrates this behavior for different TF2 and additionally indicates that specific field configurations exist which fulfill the requirements of the upgrade of the ALICE TPC. A comparison to the 4-GEM baseline configuration (S-LP-LP-S) reveals that the hybrid GEM-Micromegas detector can be considered as an alternative approach.

\section{Summary and Outlook}

A hybrid GEM-Micromegas detector consisting of two GEM foils (standard pitch) and a bulk Micromegas has been assembled at Bonn University. First measurements reveal that an energy resolution less than $12 \%(\sigma / E$ with ${ }^{55} \mathrm{Fe}$ ) and an ion backflow better than $1 \%$ is possible in $\mathrm{Ne}-\mathrm{CO}_{2}(90-10)$ at a total gain of 2000. Within the detector most of the gain is contributed by the Micromegas 


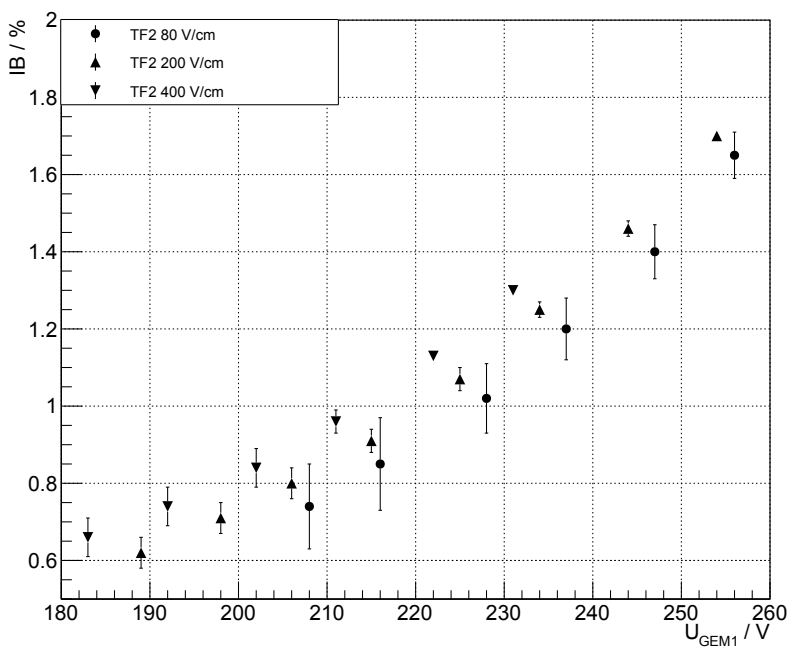

Figure 6. Ion backflow as a function of the GEM1 voltage (GEM1-MM scan in $\mathrm{Ne}-\mathrm{CO}_{2}(90-10)$, Drift $400 \mathrm{~V} / \mathrm{cm}$, TF1 $3 \mathrm{kV} / \mathrm{cm}$, GEM2 $250 \mathrm{~V}$, gain 2000)

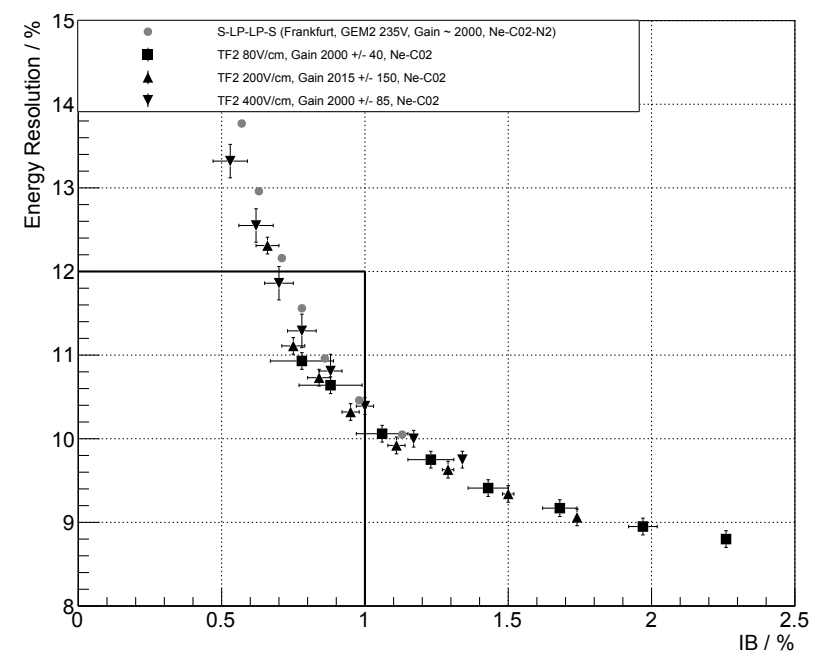

Figure 7. Energy resolution against ion backflow for the hybrid GEM-Micromegas detector (GEM1-MM scan in $\mathrm{Ne}-\mathrm{CO}_{2}$ (90-10), Drift $400 \mathrm{~V} / \mathrm{cm}$, TF1 $3 \mathrm{kV} / \mathrm{cm}$, GEM2 $250 \mathrm{~V}$, gain 2000, error energy resolution $\mathrm{x} 10$ ) and the 4-GEM baseline configuration for the ALICE TPC (Frankfurt measurements, GEM1GEM2 scan in $\mathrm{Ne}-\mathrm{CO}_{2}-\mathrm{N}_{2}(90-10-5)$ ). (order $10^{2}-10^{3}$ ), followed by the first GEM stage (order $10^{1}-10^{2}$ ). The second GEM stage is used in order to prevent ions from drifting back to the drift region while the energy resolution can be maintained. With respect to the ion backflow and the energy resolution the capability of the hybrid GEM-Micromegas detector can be compared to the 4-GEM baseline configuration and shows a similar performance. As $\mathrm{Ne}-\mathrm{CO}_{2}-\mathrm{N}_{2}$ (90-10-5) will be used for the ALICE TPC in RUN3 all previous measurements will be repeated in this gas mixture. Yet the full possible parameter space of the hybrid GEM-Micromegas detector has not been scanned in order to find an optimized configuration with respect to the ion backflow and the energy resolution. To optimize the energy resolution measurements, a veto setup for the innermost readout pads has been established and will be used in further investigations. Several measurements have shown that the quadruple S-LP-LP-S GEM configuration fulfills the specified requirements and thus has been chosen for the upgrade of the ALICE TPC. At the time of the decision the hybrid GEM-Micromegas detector was not studied as extensive as the multiple GEM setup and further stability measurements are still necessary to get a complete understanding of the hybrid GEMMicromegas detector.

\section{References}

[1] D. R. Nygren, J. N. Marx, Phys. Today 31N10, 45-53 (1978)

[2] K. H. Ackermann et al., Nucl. Phys. A 661, 681-684 (1999)

[3] ALICE Collaboration, TDR for the Upgrade of the ALICE TPC (CERN/LHCC, Geneva, 2014)

[4] G. Charpak et al., Nucl. Instr. Meth. 62, 262 (1968)

[5] B. Ketzer, Nucl. Instr. Meth. A 732, 237-240 (2013)

[6] F. Sauli, Nucl. Instr. Meth. A 386, 531-534 (1997)

[7] M. Ball et al., JINST 9, C04025 (2014)

[8] Y. Giomataris, Ph. Rebourgeard, J. P. Robert and G. Charpak, Nucl. Instr. Meth. A376, 29-35 (1996)

[9] C. Höppner, Ph.D. thesis (Tech. U., Munich, 2012)

[10] M. Killenberg et al., Nucl. Instr. Meth. A 498, 369383 (2003) 